\title{
Distflow based state estimation for power distribution networks
}

\author{
H. A. R. Florez ${ }^{1}$ • E. M. Carreno ${ }^{2}$ - M. J. Rider ${ }^{3}$ • J. R. S. Mantovani ${ }^{4}$
}

Received: 15 May 2017 / Accepted: 30 December 2017 / Published online: 15 January 2018

(C) Springer-Verlag GmbH Germany, part of Springer Nature 2018

\begin{abstract}
A new state estimation method for electrical power distribution systems using the Distflow formulation and the Weighted Least Square method to determine the steady-state operating point is presented. In order to reduce the number of measurements needed for state estimation analysis, a special set of state variables is defined. The proposed methodology is shown to be able to successfully determine the operating conditions of a electrical power distribution system with high automation levels. The proposed approach is tested on the IEEE-37 and IEEE-123 bus test system, reducing the number of state variables up to $60 \%$ when compared with conventional state estimation method.
\end{abstract}

Keywords State estimation · Electrical power distribution system · Distflow method · Weigthed least square $\cdot$ State variables

$\triangle$ H. A. R. Florez

hugoflorez@utfpr.edu.br

E. M. Carreno

emfra@ieee.org

M. J. Rider

mjrider@dsee.fee.unicamp.br

J. R. S. Mantovani

mant@dee.feis.unesp.br

1 Electrical Department, UTFPR, Medianeira, Paraná, Brazil

2 CECE-UNIOESTE, Foz do Iguaçu, Paraná, Brazil

3 Department of Systems and Energy, UNICAMP, Campinas, São Paulo, Brazil

4 Electrical Engineering Department, UNESP, Ilha Solteira, São Paulo, Brazil 


\section{List of symbols}

$\Omega L \quad$ Set of system's lines

$\Omega B \quad$ Set of system's nodes

$\Omega M$ Set of system's measurements

$\hat{x} \quad$ State variables of the system

$J(\hat{x}) \quad$ Least squares function

$z \quad$ Measurement vector

$r \quad$ Vector of measurement residuals

$h(\hat{x}) \quad$ Vector with non-linear functions

$W \quad$ Measurement weight matrix

$H$ Measurement Jacobian matrix

$G \quad$ Gain matrix

$v \quad$ Iteration counter

$Z_{i j} \quad$ Impedance of section $i j$

$R_{i j} \quad$ Resistance of section $i j$

$X_{i j} \quad$ Reactance of section $i j$

$P_{i j} \quad$ Active power flow of section $i j$

$Q_{i j} \quad$ Reactive power flow of section $i j$

$P_{i j}^{L} \quad$ Active power loss of section $i j$

$Q_{i j}^{L} \quad$ Reactive power loss of section $i j$

$V_{i}^{2} \quad$ Square of the voltage module at node $i$

$I_{i j}^{2} \quad$ Square of the current module in section $i j$

$P_{i}{ }^{D} \quad$ Active power demand at node $i$

$Q_{i}^{D} \quad$ Reactive power demand at node $i$

$P_{i}^{G} \quad$ Active power generation at node $i$

$Q_{i}^{G} \quad$ Reactive power generation at node i

$\bar{Q}_{i}^{G} \quad$ Maximum value of reactive power generation at node $i$

$Q_{i}^{G} \quad$ Minimum value of reactive power generation at node $i$

$V_{0}^{2} \quad$ Square of the voltage module at substation node

$P_{0}^{G} \quad$ Active power generation at substation node

$Q_{0}^{G} \quad$ Reactive power generation at substation node

tol Tolerance of the iterative state estimator procedure

\section{Introduction}

The real-time monitoring and control of electrical power systems (EPS) have an imperative role in power management systems (PMS). In order to evaluate the operating conditions of the system, PMS perform periodic studies of load flow and state estimation. The results are then used for safety analysis and the execution of corrective measures, so the system works within established limits.

Over the past decades, real-time analysis was exclusive to electrical power transmission systems (EPTS). Because the recent efforts to upgrade the electrical power 
distribution systems (EPDS), which involves automation and communication technologies, distributed generation (DG), Phasor Measurement Units (PMUs), among others actions, there is a need to develop new tools to better understand all the new information available.

There are several papers in the specialized literature, about conventional weight least square (WLS) state estimation in EPTS [1-3], however, those techniques could not be efficient in EPDS state estimation because there are many different characteristics. Because the operation of EPDS has reached complexity levels similar to EPTS, greater interest has been shown in developing new approaches to state estimation adapted to EPDS in recent years.

Unlike the EPTS, EPDS are larger, more complex and there are many few realtime measurements available for state estimation analysis. Therefore, in EPDS state estimation it is necessary to provide load forecasting data, which can be modeled as pseudomeasurements, to guarantee the network observability $[4,5]$.

On the conventional WLS state estimation, the module and angle for the node voltages $\left(\left|V_{k}\right|, \theta_{k}\right) \forall_{k} \in \Omega B$ are defined as state variables. Although this method is the most commonly used among the state estimators, when applied to EPDS its computational efficiency may be affected due to the high number of unknowns, large arrays involved in the process and the matrix inversion required to update the state variables. In addition, there are difficulties in this formulation to model the current meters $[6,7]$.

There are several works published on state estimation for EPTS in the specialized literature, however, almost all of these methods present several limitations when applied to EPDS, especially the high dependence on pseudo-measurements. Generally, pseudo-measurements come from load forecasting methods which depend on smooth or predictable series, which can affect the accuracy of the results because of the uncertainly levels. So, it is necessary to lower the dependence of this kind of data.

Traditionally, the EPDS state estimation has been solved mainly by the conventional node voltage state estimator, the Branch-Current State Estimator and the Branch-Power State Estimator. A short selection of the most representative methods published on the specialized literature on the last years is presented.

In Branch-Current State Estimator a special formulation developed for EPDS is used, where the real and imaginary part of the brach current $\left(I_{i j}^{r e}, I_{i j}^{i m}\right) \forall_{i j} \in \Omega L$ are defined as state variables [8-11]. In this state estimator, besides the large number of unknowns to be estimated, it is not possible to express the system quanties directly depending on the state variables. It is, therefore, necessary to transform all system measurements to branch-current magnitude measurements. The former could affect the quality of the estimated results.

On the Brach-Power Estimator proposed in $[12,13]$, the EPDS is decomposed into several feeders (single-branch) and the proposed state estimation procedure is performed separately on each feeder. Of course, the former can affect the optimal solution of the problem. Additionaly, in this approach, the authors assume that all system loads (pseudomeasurements) are known.

On the work presented on [14] state variables are the power flow on the lines $\left(P_{i j}, Q_{i j}\right)$ and the square module of the branch-currents $\left(I_{i j}^{2}\right)$. In this approach, 
the magnitudes of the system can be represented in function of the state variables as well as it is possible to incorporate a number of current meters. Moreover, the authors point out that although the number of state variables is increased, there is no need of additional measures to ensure the observability of the network. However, the success of this state estimator depends on the number of pseudomeasurements available.

To overcome these difficulties, in this paper a state estimator which defines the power injections, consumed $\left(P_{i}^{D}, Q_{i}^{D}\right)$ and injected $\left(P_{i}^{G}, Q_{i}^{G}\right)$, as state variables. The main advantage of the proposed state estimator lies in the fact of using demand forecasts as unknowns of the problem and not as essential information in order to estimate the system operating point. Moreover, considering that many of the system buses have zero power injections, the number of unknowns of the problem can be reduced considerably. To implement the proposed state estimator it is necessary to use a load flow model to calculate the system operating point as function of the power injections, which is one of the main characteristics of the Distflow formulation. This method is used in several applications in EPDS, such as optimal allocation of capacitors and DGs, reconfiguration and reduction of losses, among others [15,16]. The Distflow formulation offers the following advantages when applied in state estimation based on WLS method:

* Determining the EPDS operation point through a simplified procedure using power injections.

* Its formulation facilitates the insertion of DGs in the EPDS analyses.

* It is possible to express directly the measurement functions in terms of a special network state variable.

* It is possible to reduce the number of state variables necessary to estimate the network operation point.

* The load forecasting data is used only to start the state variables. Thus, the influence of the pseudomeasurements on the estimated results can be reduced when the number of real-time measurements is increased.

In the next sections, a state estimator for EPDS based on an adaptation of the Distflow sweep formulation and WLS methods for determining the operating point of the system is presented.

To validate the methodology, the networks operating point for a given scenario is calculated using the Distflow method. Subsequently, the results of the power flow analysis are used to create a measurement set to be used as input information. After, adding normal random Gaussian errors to the measurement set, the operating point of the EPDS is calculated using the proposed state estimator. In this way, the validation process is based on a comparison of the operating points obtained using both approaches and through the WLS function obtained from the simulations.

\section{Distflow method}

The Distflow method was developed for load flow analysis in radial or weakly meshed electrical systems. There are two versions of the Distflow method in the specialized 


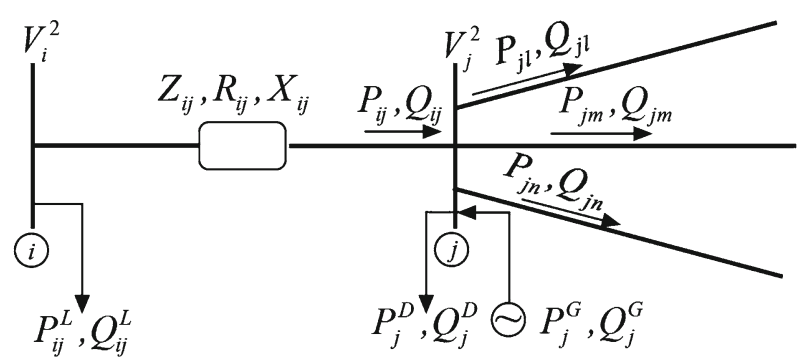

Fig. 1 Magnitudes downstream of section $i j$ of an EPDS

literature. In the original version, it is necessary to carry out an iterative process that involves performing a downstream sweep and solving a nonlinear equation system using Newton's method to determine the operation point of the system [17]. On the other hand, a Backward/Forward version of the Distflow method is proposed in [18], where the magnitudes of the variables in the system are updated by sweeping in both directions instead of solving the system of nonlinear equations. This sweep version is simpler and more advantageous because it does not require large matrices or matrix inversion procedures to solve the system of nonlinear equations.

\subsection{Formulation of the distflow method}

For simplicity and for the purpose of state estimation, a different formulation of the Distflow method is used, rather than the ones presented in $[17,18]$. Thus, the Backward/Forward version of the Distflow method uses a set of equations for each section $i j$ of the network, which represents the steady-state operation of an EPDS that takes into account the presence of DGs.

According to Fig. 1, in the backward sweep the variables $P_{i j}, Q_{i j}$ of section $i j$ may be determined as follows:

$$
\begin{aligned}
P_{i j} & =\sum_{j l \in \Omega L}\left(P_{j l}+P_{j l}^{L}\right)-\sum_{\substack{m j \in \Omega L \\
m \neq i}} P_{m j}+P_{j}^{D}-P_{j}^{G} \\
Q_{i j} & =\sum_{j l \in \Omega L}\left(Q_{j l}+Q_{j l}^{L}\right)-\sum_{\substack{m j \in \Omega L \\
m \neq i}} Q_{m j}+Q_{j}^{D}-Q_{j}^{G}
\end{aligned}
$$

Where the power losses in section $j l$ are given by:

$$
\begin{aligned}
& P_{j l}^{L}=R_{j l}\left(\frac{P_{j l}^{2}+Q_{j l}^{2}}{V_{l}^{2}}\right) \\
& Q_{j l}^{L}=X_{j l}\left(\frac{P_{j l}^{2}+Q_{j l}^{2}}{V_{l}^{2}}\right)
\end{aligned}
$$


On the other hand, in the forward sweep the variable $V_{j}$ can be calculated as follows::

$$
V_{j}^{2}=V_{i}^{2}-2\left(R_{i j} P_{i j}+X_{i j} Q_{i j}\right)+Z_{i j}^{2}\left(\frac{P_{i j}^{2}+Q_{i j}^{2}}{V_{i}^{2}}\right)
$$

Therefore, for an EPDS with $\mathrm{n}$ nodes and $(n-1)$ sections the Distflow method uses $3(n-1)$ equations to estimate $2(n-1)$ power flows and $(n-1)$ voltage magnitudes, assuming the substation bus voltage and the power injections are known.

\section{Proposed state estimation}

In conventional state estimation analysis, the WLS method seeks to minimize the effect of measurement errors on the estimated results. Thus, the state estimator determines the values of $\hat{x}$ that minimize the following quadratic function [6]:

$$
\begin{aligned}
\text { minimize } \quad J(\hat{x}) & =r^{t} W r \\
\text { subject to }: \quad r_{m} & =z_{m}-h_{m}(\hat{x}) \forall_{m} \in \Omega M
\end{aligned}
$$

Where $W$ is a diagonal matrix whose values assigned to each measurement reflect their accuracy. The quadratic problem shown in (6) can be solved as an unconstrained minimization problem by an iterative numerical procedure. Thus, to find the vector $\hat{x}$ that minimizes $J(\hat{x})$, the first-order optimality conditions will have to be satisfied as shown below:

$$
\frac{\partial J(\hat{x})}{\partial \hat{x}}=H^{t} W(z-h(\hat{x}))=0
$$

The solution of the above equations is achieved by Newton's iterative method according to the following expression:

$$
\hat{x}^{(v+1)}=\hat{x}^{(v)}+G^{-1} H^{t} W\left(z-h\left(\hat{x}^{(v)}\right)\right)
$$

Where the matrix $G=H^{t} W H$ is sparse, symmetric and positive definite for fully observable networks. In the conventional state estimator, once the values of $\hat{x}$ are estimated, a second evaluation phase begins with the results obtained through the processing of significant errors $[19,20]$. These procedures seek to identify and correct erroneous measurements such that the results provided by the state estimator are reliable. Despite their importance, the handling of errors is outside the scope of the present work.

The proposed state estimator calculates the operating point of the EPDS through the Backward/Forward version of the Distflow method along with the Newton's iterative method used in the conventional WLS state estimation to solve (8). Therefore, to develop the proposed state estimator, it is necessary to consider the following aspects: 


\subsection{Definition of State Variables $\hat{x}$}

According to the equations of the Backward/Forward version of the Distflow method, for an EPDS with n nodes and $(n-1)$ sections, there will be $3(n-1)$ equations and $5 n-4$ variables to estimate: $2(n-1)$ power flows, $n$ voltage magnitudes and $4(n-1)$ power injections. Taking into account that the state variables represent the minimum set of variables necessary to determine the operating point of the system, the following set of state variables is introduced:

* The slack bus voltage $\left(V_{0}^{2}\right)$.

* The active and reactive power injections $\left(P_{k}^{G}, Q_{k}^{G}, P_{k}^{D}, Q_{k}^{D}\right) \forall_{k} \in \Omega B$.

The selection of this set of state variables can reduce the number of unknown variables; in EPDS, there are a large number of nodes with no power injection, known as zero-injection buses.

\subsection{Measurement functions $h(\hat{x})$}

From the Distflow formulation shown in (1-5), it is possible to develop a measurement set for active and reactive power flows injections and for absolute voltage values. Additionally, through the following expressions, one can incorporate the current values into the Distflow Backward/Forward sweep:

$$
I_{i j}^{2}=\frac{P_{i j}^{2}+Q_{i j}^{2}}{V_{i}^{2}}
$$

Thus, the main types of measurements provided to the estimator are listed below:

* The substation voltage and the power injections at the substation.

* Voltage, current and power flow at specific system location.

* Power demand provided by load forecasting.

* Voltage and power injection in nodes containing DGs.

These measurements can be estimed in terms of the proposed state variables, once backward/forward sweep is executed. The number and location of measurements must ensure the observability of the network.

\subsection{Structure of Jacobian matrix}

The matrix $H$ for the proposed state estimator presents the following structure: 


$$
\begin{aligned}
& P_{m}^{G} Q_{m}^{G} \ldots P_{n}^{G} \quad Q_{n}^{G} \quad P_{m}^{D} \quad Q_{m}^{D} \ldots P_{n}^{D} \quad Q_{n}^{D}
\end{aligned}
$$

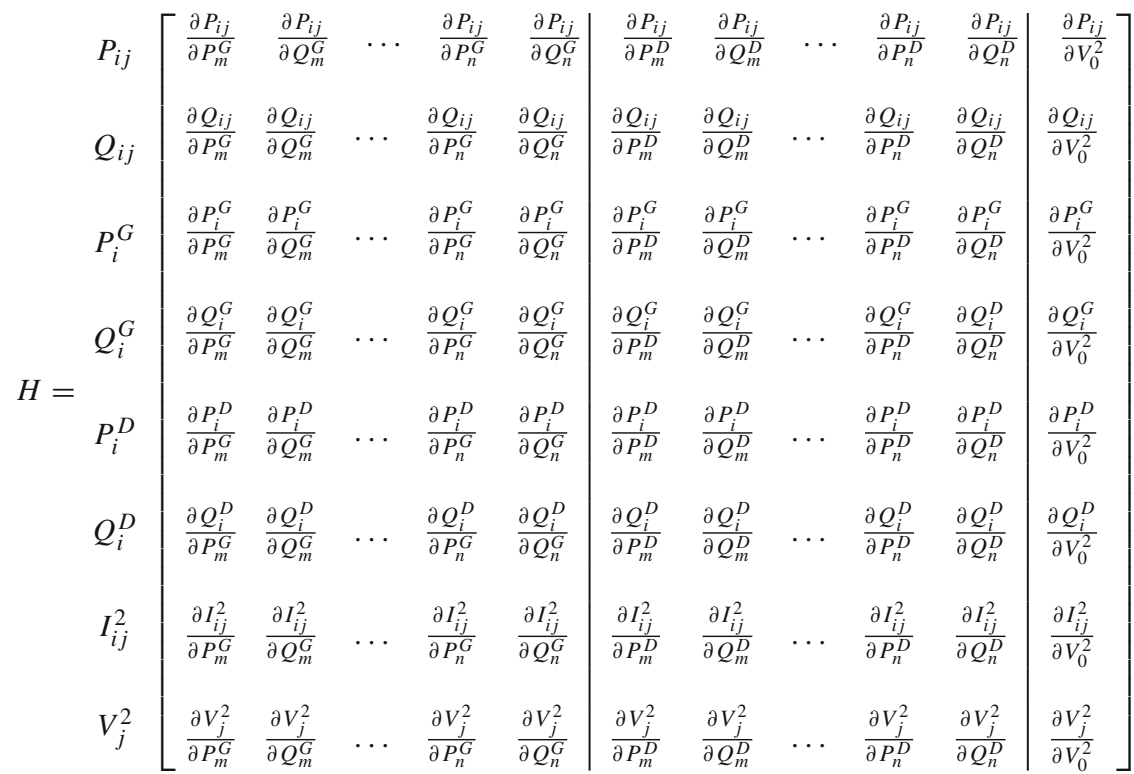

Similar to the strategy adopted in [17], the appendix shows that, through a series of approximations, it is possible to easily obtain a matrix $H$. These approximations, although occurring during the iterative process of the algorithm, allow for the quick and efficient estimation of the operating point of the system.

\subsection{Algorithm of the proposed state estimator}

To estimate the operating point of the system we propose the following procedure based on the WLS and Distflow method:

i. Input data System parameter values. Measurement vector $z$ and their weights $W, \forall_{m} \in \Omega M$.

ii. Initial values of $\hat{x}$ The initial values of the state variables can be defined according to the values measured at the substation, such as the substation voltage and the power demanded by the EPDS. Thus, we assume that $V_{0}^{2}=1$ in the slack bus, $P_{i}^{D}$ and $Q_{i}^{D}$ is divided proportionally among all nodes in the network and $P_{i}^{G}=0$ and $Q_{i}^{G}=0$ in the PV nodes.

iii. Start of the iterative process Initialize the iteration counter $(v=0)$ and set the nodal voltages equals to slack bus voltage. Go to step iv.

iv. Distflow backward sweep For each section $i j$, calculate $P_{i j}^{(v)}, Q_{i j}^{(v)}$ using $(1,2)$. Go to step v.

v. Distflow Forward sweep. For each section $i j$ calculate $V_{j}^{2^{(v)}}$ using (5) and $I_{i j}^{2^{(v)}}$ using (9). 
Table 1 DGs parameters in p.u.

\begin{tabular}{llllll}
\hline System & $i$ & $P_{i}^{G}$ & $V_{i}^{s c h}$ & $\underline{Q}_{i}^{G}$ & $\bar{Q}_{i}^{G}$ \\
\hline IEEE-37 & 735 & 0.0500 & 0.9234 & -0.0250 & 0.0300 \\
& 740 & 0.0750 & 0.9202 & -0.0250 & 0.0500 \\
IEEE-123 & 66 & 0.0500 & 0.9044 & -0.0250 & 0.0300 \\
& 96 & 0.0900 & 0.9005 & -0.0500 & 0.0700 \\
& 114 & 0.1000 & 0.9005 & -0.0500 & 0.0700 \\
\hline
\end{tabular}

vi. Calculate the measurements residuals The estimated measurements and their residual vector are computed using $r_{m}=z_{m}-h_{m}\left(\hat{x}^{(v)}\right), \forall_{m} \in \Omega M$.

vii. Update $\hat{x}^{(v)}$ values. The values of $\hat{x}^{(v)}$ are updated using (8). Set $v=v+1$ and go to step viii.

viii. Stop criteria If $\left|\hat{x}^{(v)}-\hat{x}^{(v-1)}\right| \leq t o l$, go to step ix. Otherwise, go back to step iv.

\section{ix. Output results.}

\section{Test and result}

The proposed state estimator for EPDS was run in the software Matlab and was tested on IEEE-37 and IEEE-123 test systems on a Dell computer with an Intel Core i7 processor and $4 \mathrm{~GB}$ of RAM.

During the simulations, the test systems were overloaded with the intent of building scenarios that contain DGs. Among the main considerations in determining the operating point of the test system, the following stand out:

* Voltage regulators, transformers, switches and capacitor banks are not taken into account.

* Load bars are represented by a constant power model.

* The series impedance values of the lines were multiplied by a factor of $K_{L}=10$.

* The base voltage is assumed to be $12.66 \mathrm{kV}$ and the base power, $1.0 \mathrm{MW}$.

In order to create the measurement set, Gaussian errors are inserted in the voltage, current and power flow values obtained in the load flow analysis of the Distflow method. Additionally, some nodal power demands are used as pseudo-measurements. In both test systems, the weight of the real-time measurements and the pseudomeasurements are 0.16 and 0.04 , respectively.

The proposed state estimator was validated using the information contained in [21]. Thus, taking as reference the values from phases $\mathrm{C}$ and $\mathrm{A}$ of the IEEE-37 and IEEE123 systems, the operating point was calculated using the Distflow method. Table 1 shows the data from the DGs used in the simulations.

The operating point of the system given by these input values is used as a basis to create the measurement set. However, it is first necessary to identify the number of zero injection buses that are present in the system to determine the number of state variables. In Table 2, it is shown that, through the proposed state estimator, it is possible 
Table 2 Number of state variables to be estimated

Table 3 Performance of the proposed state estimator

\begin{tabular}{lll}
\hline System & Conventional Estimator & Proposed Estimator \\
\hline IEEE-37 & 73 & 27 \\
IEEE-123 & 245 & 83 \\
\hline
\end{tabular}

\begin{tabular}{llll}
\hline System & $J(\hat{x})$ & Number of Iterations & Execution time (s) \\
\hline IEEE-37 & 4.2987 & 4 & 0.0564 \\
IEEE-123 & 7.1611 & 5 & 0.0964 \\
\hline
\end{tabular}

Table 4 Maximum estimated erros in p.u.

\begin{tabular}{lllll}
\hline System & $\hat{x}$ & Real value & Estimated value & $\mid$ Error $\mid$ \\
\hline IEEE-37 & $P_{701}^{D}$ & 0.3500 & 0.3443 & 0.0057 \\
& $Q_{712}^{D}$ & 0.0850 & 0.0895 & 0.0045 \\
& $Q_{735}^{G}$ & 0.0199 & 0.0242 & 0.0043 \\
& $Q_{701}^{D}$ & 0.1750 & 0.1718 & 0.0032 \\
& $V_{799}^{2}$ & 1.0000 & 1.0027 & 0.0027 \\
& $P_{735}^{G}$ & 0.0500 & 0.0478 & 0.0022 \\
& $Q_{76}^{D}$ & 0.0800 & 0.0739 & 0.0061 \\
& $P_{76}^{D}$ & 0.1050 & 0.1109 & 0.0059 \\
& $P_{48}^{D}$ & 0.0700 & 0.0644 & 0.0056 \\
& $Q_{55}^{D}$ & 0.0200 & 0.0243 & 0.0043 \\
& $Q_{48}^{D}$ & 0.0500 & 0.0461 & 0.0039 \\
& $Q_{66}^{G}$ & 0.0249 & 0.0281 & 0.0032 \\
\hline
\end{tabular}

to reduce the number of unknown variables when compared to the conventional state estimator.

Considering that the network observability depends on the number, type and location of the meters, a measurement set composed by several meters distributed throughout the system was created, which makes the system observable. The performance of the proposed algorithm is presented in Table 3, assuming a convergence criterion of $t o l=10^{-4}$.

These values show that the proposed algorithm rapidly converges. Furthermore, the $J(\hat{x})$ values indicate that the operating point of the system was calculated satisfactorily, according to the hypothesis tests of the conventional state estimator. Table 4 shows the estimated maximum residues of $\hat{x}$ for both test systems.

Finally, considering the load flow results as the real operating point of the system, the power losses, as well as the voltage magnitudes and the power levels generated at the substation, obtained through the load flow and the proposed state estimator are compared in the Table 5. 
Table 5 Comparison between real and estimated operation point

\begin{tabular}{llllll}
\hline & Real value & & & \multicolumn{2}{c}{ Estimated value } \\
\cline { 2 - 3 } & IEEE-37 & IEEE-123 & & IEEE-37 & IEEE-123 \\
\hline$P^{L}$ & 0.0455 & 0.0646 & & 0.0523 & 0.0598 \\
$P_{0}^{G}$ & 1.0115 & 1.2446 & & 1.0068 & 1.2511 \\
$Q_{0}^{G}$ & 0.5204 & 0.7641 & & 0.5177 & 0.7738 \\
$V_{0}^{2}$ & 1.0000 & 1.0000 & & 1.0015 & 1.0022 \\
\hline
\end{tabular}

From the above results, it can be observed that the estimated results are very close to the real values obtained by load flow analysis. This confirms that proposed state estimator is able to obtain satisfactory results when applied to EPDS.

\section{Discussion and conclusions}

This article presents a novel state estimator that uses the WLS and the Distflow formulation to evaluate the operating point of the system. The main contribution is a reduced set of state variables.

The proposed methodology was validated by two IEEE test systems. These results show that through a simplified procedure, the Distflow based state estimator can be used to estimate successfully the EPDS operation point. Additionally, we show that because of the special formulation it is possible to reduce the number of state variables up of $65 \%$ on the test systems. Clearly, this condition represents an advantage for gross errors analyses in EPDS state estimation, because the measurement redundancy levels are incremented.

This new approach is an important contribution to the field of state estimation for EPDS because it was able to solve efficiently some limiting factors present in other state estimator methodologies. Therefore, the proposed methodology proves it could be a promising tool for future research dealing with automation and control of modern EPDS.

Future research could include adopting a gross errors analysis according to the characteristics of the EPDS. Furthermore, an unbalanced, three-phase version of the state estimator should be developed.

\section{A Structure of Jacobian matrix $\boldsymbol{H}$}

In the same way that the backward sweep formulation shown in (1-2), the power flows $P_{i j}$ and $Q_{i j}$ can be calculated as show in Fig. 2:

$$
P_{i j}=\sum_{l i \in \Omega L}\left(P_{l i}-P_{l i}^{L}\right)-\sum_{\substack{i m \in \Omega L \\ m \neq j}} P_{i m}-P_{i}^{D}+P_{i}^{G}
$$




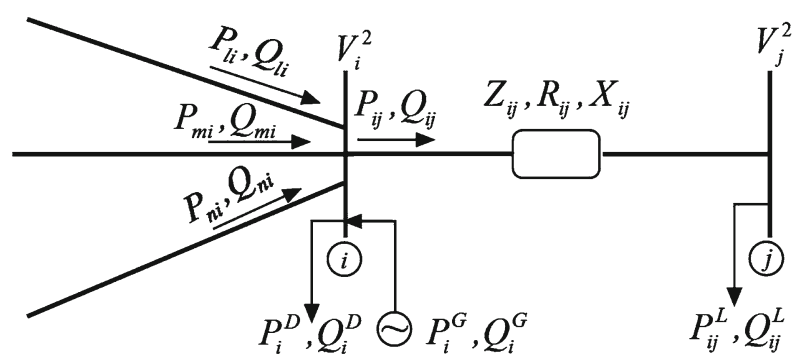

Fig. 2 Magnitudes upstream of section $i j$ of an EPDS

$$
Q_{i j}=\sum_{l i \in \Omega L}\left(Q_{l i}-Q_{l i}^{L}\right)-\sum_{\substack{i m \in \Omega L \\ m \neq j}} Q_{i m}-Q_{i}^{D}+Q_{i}^{G}
$$

where,

$$
\begin{aligned}
& P_{l i}^{L}=R_{l i}\left(\frac{P_{l i}^{2}+Q_{l i}^{2}}{V_{l}^{2}}\right) \\
& Q_{l i}^{L}=X_{l i}\left(\frac{P_{l i}^{2}+Q_{l i}^{2}}{V_{l}^{2}}\right)
\end{aligned}
$$

Thus, according to the Backward/Forward Distflow formulation, it is possible to obtain the following terms from $H(\hat{x})$ directly:

$$
\begin{aligned}
& \frac{\partial P_{i j}}{\partial P_{i}^{G}}=\frac{\partial Q_{i j}}{\partial Q_{i}^{G}}=\frac{\partial P_{i j}}{\partial P_{j}^{D}}=\frac{\partial Q_{i j}}{\partial Q_{j}^{D}}=1 \\
& \frac{\partial P_{i j}}{\partial P_{j}^{G}}=\frac{\partial Q_{i j}}{\partial Q_{j}^{G}}=\frac{\partial P_{i j}}{\partial P_{i}^{D}}=\frac{\partial Q_{i j}}{\partial Q_{i}^{D}}=-1 \\
& \frac{\partial P_{i j}}{\partial Q_{i}^{G}}=\frac{\partial Q_{i j}}{\partial P_{i}^{G}}=\frac{\partial P_{i j}}{\partial Q_{j}^{G}}=\frac{\partial Q_{i j}}{\partial P_{j}^{G}}=0 \\
& \frac{\partial P_{i j}}{\partial Q_{i}^{D}}=\frac{\partial Q_{i j}}{\partial P_{i}^{D}}=\frac{\partial P_{i j}}{\partial Q_{j}^{D}}=\frac{\partial Q_{i j}}{\partial P_{j}^{D}}=0
\end{aligned}
$$

However, due to the lack of equations that relate the magnitudes to the state variables of the system, the chain rule is used to determine the elements of $H$ [17].

\section{A.1 Voltage measurements}

The elements of $H$ correspondent to the measured voltage $V_{j}^{2}$ are calculated as shown below.

$$
\frac{\partial V_{j}^{2}}{\partial V_{0}^{2}}=\left(\frac{\partial V_{j}^{2}}{\partial V_{i}^{2}}\right)\left(\frac{\partial V_{i}^{2}}{\partial V_{(i-1)}^{2}}\right) \cdots\left(\frac{\partial V_{(k+1)}^{2}}{\partial V_{0}^{2}}\right)
$$




$$
\begin{aligned}
\frac{\partial V_{j}^{2}}{\partial P_{m}^{G}}=\left(\frac{\partial V_{j}^{2}}{\partial P_{i j}}\right)\left(\frac{\partial P_{i j}}{\partial P_{(i-1) i}}\right) \cdots\left(\frac{\partial P_{m(m+1)}}{\partial P_{m}^{G}}\right) & \partial V_{j}^{2} \\
\frac{\partial P_{m}^{D}}{\partial V_{j}^{2}} & =\left(\frac{\partial P_{i j}}{\partial P_{(i-1) i}}\right) \cdots\left(\frac{\partial P_{m(m+1)}}{\partial P_{m}^{D}}\right) \\
\frac{\partial V_{j}^{2}}{\partial Q_{m}^{G}} & =\left(\frac{\partial V_{j}^{2}}{\partial Q_{i j}}\right)\left(\frac{\partial Q_{i j}}{\partial Q_{(i-1) i}}\right) \cdots\left(\frac{\partial Q_{m(m+1)}}{\partial Q_{m}^{G}}\right) \\
\frac{\partial V_{j}^{2}}{\partial Q_{m}^{D}} & =\left(\frac{\partial V_{j}^{2}}{\partial Q_{i j}}\right)\left(\frac{\partial Q_{i j}}{\partial Q_{(i-1) i}}\right) \cdots\left(\frac{\partial Q_{m(m+1)}}{\partial Q_{m}^{D}}\right) \\
\frac{\partial V_{j}^{2}}{\partial P_{n}^{G}} & =\left(\frac{\partial V_{j}^{2}}{\partial P_{i j}}\right)\left(\frac{\partial P_{i j}}{\partial P_{j(j+1)}}\right) \cdots\left(\frac{\partial P_{(n-1) n}}{\partial P_{n}^{G}}\right) \\
\frac{\partial V_{j}^{2}}{\partial P_{n}^{D}} & =\left(\frac{\partial V_{j}^{2}}{\partial P_{i j}}\right)\left(\frac{\partial P_{i j}}{\partial P_{j(j+1)}}\right) \cdots\left(\frac{\partial P_{(n-1) n}}{\partial P_{n}^{D}}\right) \\
\frac{\partial V_{j}^{2}}{\partial Q_{n}^{G}} & =\left(\frac{\partial V_{j}^{2}}{\partial Q_{i j}}\right)\left(\frac{\partial Q_{i j}}{\partial Q_{j(j+1)}}\right) \cdots\left(\frac{\partial Q_{(n-1) n}}{\partial Q_{n}^{G}}\right) \\
\frac{\partial V_{j}^{2}}{\partial Q_{n}^{D}} & =\left(\frac{\partial V_{j}^{2}}{\partial Q_{i j}}\right)\left(\frac{\partial Q_{i j}}{\partial Q_{j(j+1)}}\right) \cdots\left(\frac{\partial Q_{(n-1) n}}{\partial Q_{n}^{D}}\right)
\end{aligned}
$$

Therefore, the high computational effort necessary to evaluate the elements of $H$ for each section of the system is clear. This disadvantage can be mitigated as follows:

* Considering that in per unit $Z_{i j}^{2} \ll 1, \forall_{i j} \in \Omega L$, we have:

$$
\begin{aligned}
& \frac{\partial V_{j}^{2}}{\partial V_{i}^{2}}=1+Z_{i j}^{2}\left(\frac{P_{i j}^{2}+Q_{i j}^{2}}{V_{i}^{4}}\right) \approx 1 \\
& \frac{\partial V_{j}^{2}}{\partial P_{i j}}=-2 R_{i j}+2 P_{i j} Z_{i j}^{2} \approx-2 R_{i j} \\
& \frac{\partial V_{j}^{2}}{\partial Q_{i j}}=-2 X_{i j}+2 Q_{i j} Z_{i j}^{2} \approx-2 X_{i j}
\end{aligned}
$$

* Considering that in per unit the terms: $R_{i j} \frac{P_{i j}}{V_{i}^{2}} \ll 1, R_{i j} \frac{P_{i j}}{V_{j}^{2}} \ll 1, X_{i j} \frac{Q_{i j}}{V_{i}^{2}} \ll 1$ and $X_{i j} \frac{Q_{i j}}{V_{j}^{2}} \ll 1, \forall_{i j} \in \Omega L$, we have:

$$
\begin{gathered}
\frac{\partial P_{i j}}{\partial P_{j(j+1)}}=1+2 R_{j(j+1)}\left(\frac{P_{j(j+1)}}{V_{j+1}^{2}}\right) \approx 1 \\
\frac{\partial P_{i j}}{\partial P_{(i-1) i}}=1-2 R_{(i-1) i}\left(\frac{P_{(i-1) i}}{V_{i-1}^{2}}\right) \approx 1
\end{gathered}
$$




$$
\begin{gathered}
\frac{\partial Q_{i j}}{\partial Q_{j(j+1)}}=1+2 X_{j(j+1)}\left(\frac{Q_{j(j+1)}}{V_{j+1}^{2}}\right) \approx 1 \\
\frac{\partial Q_{i j}}{\partial Q_{(i-1) i}}=1-2 X_{(i-1) i}\left(\frac{Q_{(i-1) i}}{V_{i-1}^{2}}\right) \approx 1
\end{gathered}
$$

Thus, the elements of $H$ shown in (19-27) are calculated as follows:

$$
\begin{gathered}
\frac{\partial V_{j}^{2}}{\partial V_{0}^{2}} \approx 1 \\
\frac{\partial V_{j}^{2}}{\partial P_{m}^{G}}=-\frac{\partial V_{i}^{2}}{\partial P_{m}^{D}} \approx 2 R_{i j} \\
\frac{\partial V_{j}^{2}}{\partial Q_{m}^{G}}=-\frac{\partial V_{i}^{2}}{\partial Q_{m}^{D}} \approx 2 X_{i j} \\
\frac{\partial V_{j}^{2}}{\partial P_{n}^{G}}=-\frac{\partial V_{i}^{2}}{\partial P_{n}^{D}} \approx-2 R_{i j} \\
\frac{\partial V_{j}^{2}}{\partial Q_{n}^{G}}=-\frac{\partial V_{i}^{2}}{\partial Q_{n}^{D}} \approx-2 X_{i j}
\end{gathered}
$$

\section{A.2 Power flow measurements}

Considering that in per unit the terms $R_{i j}\left(\frac{P_{i j}^{2}+Q_{i j}^{2}}{V_{i}^{4}}\right) \ll 1$, e $X_{i j}\left(\frac{P_{i} j^{2}+Q_{i} j^{2}}{V_{i}^{4}}\right) \ll 1$, we have:

$$
\begin{aligned}
& \frac{\partial P_{i j}}{\partial V_{0}^{2}}=R_{i(i-1)}\left(\frac{\partial P_{i(i-1)}^{2}+Q_{i(i-1)}^{2}}{\partial V_{(i-1)}^{4}}\right) \approx 0 \\
& \frac{\partial Q_{i j}}{\partial V_{0}^{2}}=X_{i(i-1)}\left(\frac{\partial P_{i(i-1)}^{2}+Q_{i(i-1)}^{2}}{\partial V_{(i-1)}^{4}}\right) \approx 0
\end{aligned}
$$

\section{A.3 Power injection measurements}

$$
\begin{gathered}
\frac{\partial P_{i}^{G}}{\partial P_{m}^{G}}=\frac{\partial Q_{i}^{G}}{\partial Q_{m}^{G}}=\frac{\partial P_{i}^{D}}{\partial P_{m}^{D}}=\frac{\partial Q_{i}^{D}}{\partial Q_{m}^{D}}=\left\{\begin{array}{c}
1, \text { if } i=m \\
0, \text { if } i \neq m
\end{array}\right. \\
\frac{\partial P_{i}^{G}}{\partial Q_{m}^{G}}=\frac{\partial P_{i}^{G}}{\partial Q_{m}^{D}}=\frac{\partial P_{i}^{G}}{\partial V_{0}^{2}}=\frac{\partial Q_{i}^{G}}{\partial P_{m}^{G}}=\frac{\partial Q_{i}^{G}}{\partial P_{m}^{D}}=\frac{\partial Q_{i}^{G}}{\partial V_{0}^{2}}=0
\end{gathered}
$$




$$
\frac{\partial P_{i}^{D}}{\partial Q_{m}^{G}}=\frac{\partial P_{i}^{D}}{\partial Q_{m}^{D}}=\frac{\partial P_{i}^{D}}{\partial V_{0}^{2}}=\frac{\partial Q_{i}^{D}}{\partial P_{m}^{G}}=\frac{\partial Q_{i}^{D}}{\partial P_{m}^{D}}=\frac{\partial Q_{i}^{D}}{\partial V_{0}^{2}}=0
$$

\section{A.4 Current measurements}

$$
\begin{aligned}
\frac{\partial I_{i j}^{2}}{\partial P_{m}^{G}} & =-\frac{\partial I_{i j}^{2}}{\partial P_{m}^{D}} \approx \frac{2 P_{i j}}{V_{j}} \\
\frac{\partial I_{i j}^{2}}{\partial Q_{m}^{G}} & =-\frac{\partial I_{i j}^{2}}{\partial Q_{m}^{D}} \approx \frac{2 Q_{i j}}{V_{j}} \\
\frac{\partial I_{i j}^{2}}{\partial P_{n}^{G}} & =-\frac{\partial I_{i j}^{2}}{\partial P_{n}^{D}} \approx-\frac{2 P_{i j}}{V_{j}} \\
\frac{\partial I_{i j}^{2}}{\partial Q_{n}^{G}} & =-\frac{\partial I_{i j}^{2}}{\partial Q_{n}^{D}} \approx-\frac{2 Q_{i j}}{V_{j}} \\
\frac{\partial I_{i j}^{2}}{\partial V_{0}^{2}} & \approx \frac{P_{i j}^{2}+Q_{i j}^{2}}{V_{i}^{2}}
\end{aligned}
$$

\section{References}

1. Coutto, M., Leite, A., Falco, D.: Bibliography on power system state estimation (1968-1989). IEEE Trans. Power Syst. 5, 950-961 (1990)

2. Monticelli, A.: State Estimation in Electric Power Systems. Kluwer Academic Publishers, Boston (1999)

3. Grainger, J.J., Stevenson, W.D.: Power System Analysis. Mc Graw Hill, New York (1994)

4. Lin, W.H., Teng, J.H., Liu, W.H.: State estimation for distribution systems with zero-injection constraints. IEEE Trans. Power Syst. 11, 518524 (1996)

5. Singh, R., Pal, B.C., Jabr, R.A.: Distribution system state estimation through Gaussian mixture model of the load as pseudo-measurements. IET Gen. Trans. Distrib. 4, 50-59 (2010)

6. Abur, A., Expsito, A.G.: Power System State Estimation: Theory and Implementation. Marcel Dekker, New York (2004)

7. Lu, C.N., Teng, J.H., Liu, W.H.: Distribution system state estimation. IEEE Trans. Power Syst. 10, 229240 (1995)

8. Baran, M.E., Kelley, A.W.: A branch-current-based state estimation method for distribution systems. IEEE Trans. Power Syst. 10, 483491 (1995)

9. Wang, H., Schulz, N.N.: A revised branch current-based distribution system state estimation algorithm and meter placement impact. IEEE Trans. Power Syst. 19, 207213 (2004)

10. Baran, M. E., Jung, J., McDermott, T.: Including voltage measurements in branch-current state estimation for distribution systems. In: IEEE Proceedings of the Power Energy Society General Meeting (2009). https://doi.org/10.1109/PES.2009.5275479

11. Pau, M., Pegoraro, P.A., Sulis, S.: Efficient branch-current-based distribution system state estimation including synchronized measurements. IEEE Trans. Implement Measur 62, 24192429 (2013)

12. Sun, H., Zhang, B., Xiang, N.: A branch-power-based state estimation method for distribution systems. Autom. Electr. Power Syst. 22, 2-17 (1998)

13. Deng, Y., He, Y., Zhang, B.: A branch-estimation-based state estimation method for radial distribution systems. IEEE Trans. Power Deliv. 17, 1057-1062 (2002)

14. Wenchuan, W., Yuntao, J., Boming, Z., Hongbin, S.: A distribution system state estimator accommodating large number of ampere measurements. Int. J. Electr. Power Energy Syst. 43, 839-848 (2012) 
15. Baran, M.E., Wu, F.F.: Optimal capacitors placement on a radial distribution systems. IEEE Trans. Power Deliv. 4, 725734 (1989)

16. Franco, F., Rider, M., Romero, R.: A mixed-integer quadratically-constrained programming model for the distribution system expansion planning. Int. J. Electr. Power Energy Syst. 62, 265-272 (2014)

17. Baran, M.E., Wu, F.F.: Optimal sizing of capacitors placed on a radial distribution system. IEEE Trans. Power Deliv. 4, 735743 (1989)

18. Rao, R.S., Narasimham, S., Ramalingaraju, M.: Optimization of distribution network configuration for loss reduction using artificial bee colony algorithm. Int. J. Electr. Power Energy Syst. Eng. 1, 116122 (2008)

19. Mili, L., Van Cutsen, T.: Implementation of the hypothesis testing identification in power system state estimation. IEEE Trans. Power Syst. 3, 887893 (1988)

20. Mili, L., Van Cutsen, T., Ribbens-Pavella, M.: Bad data identification methods in power system state estimation: a comparative study. IEEE Trans. Power Appar. Syst. PAS-104, 3037-3049 (1985).

21. Kersting, W. H.: Radial distribution test feeders. In: Proceedings of the IEEE Power Engineering Society Winter Meeting (2001). https://doi.org/10.1109/PESW.2001.916993 